\title{
Ketoprofen induced photoallergic reaction
}

\author{
Authors: $\underline{\text { Rosan } \mathrm{T}^{1}}$, Ljubojević Hadžavdić $\mathrm{S}^{1,2}$ \\ ${ }^{1}$ School of Medicine, University of Zagreb, Zagreb, Croatia \\ ${ }^{2}$ Department of Dermatology and Venereology, University Hospital Center Zagreb, Zagreb, Croatia \\ DOI: https://doi.org/10.26800/LV-142-supp5-31
}

\section{Background:}

Photoallergic reactions are hypersensitivity reactions which occur when ultraviolet radiation interacts with ingredients in drugs or other products applied directly to the skin. The body's immune system recognizes the changes caused by ultraviolet radiation as a foreign threat, so it produces antibodies and causes inflammation of the skin in the exposed areas. Common photoallergic drugs and ingredients are included in some sunscreens, aftershave lotions, antimicrobials (especially sulfonamides), non-steroidal anti-inflammatory drugs (NSAIDs), chemotherapy drugs and fragrances.

\section{Case presentation:}

A 64-year-old female patient was admitted to the Department of Dermatology and Venereology with erythema and underlining edema on her left foot. A few weeks before the patient had a fracture of the metatarsal bones and since then she has been taking NSAIDs systemically to suppress pain. Five days before being admitted to the hospital she started applying Fastum ${ }^{\circledR}$ (ketoprofen) gel to her left foot twice daily. She was frequently exposed to the sun. For the last twenty years she has been struggling with chronic back pain and frequently taking different NSAIDs (ibuprofen, diclofenac, etc). She also suffers from essential hypertension and regularly takes ramipril (ACE inhibitor). The photopatch test administered alongside topical ketoprofen was positive, confirming diagnosis. She was advised to discontinue ketoprofen application and to instead apply bethamethason cream twice daily for 7 days.

\section{Conclusion:}

Ketoprofen is a NSAID that is commonly used both topically and systemically for treatment of musculoskeletal diseases because of its analgesic and anti-inflammatory effects. Ketoprofen induced photosensitivity reactions usually present as photoallergic dermatitis, which is a cell-mediated immune process.

Keywords: ketoprofen, photoallergic dermatitis, photoallergic reaction 